\title{
The barrier to linearity of hydrogen sulphide
}

\author{
György Tarczay a , Attila G. Császár a, * , Matthew L. Leininger ${ }^{\text {b }}$, Wim Klopper c \\ a Department of Theoretical Chemistry, Eötvös University, P.O. Box 32, H-1518 Budapest 112, Hungary \\ ${ }^{\mathrm{b}}$ Sandia National Laboratory, MS 9214, Livermore, CA 94551-0969, USA \\ c Theoretical Chemistry Group, Debye Institute, Utrecht University, Padualaan 14, NL-3584 CH Utrecht, The Netherlands
}

Received 23 February 2000; in final form 27 March 2000

\begin{abstract}
High-quality ab initio quantum chemical methods, including higher-order coupled cluster (CC) and many-body perturbation (MP) theory, as well as full configuration interaction (FCI) benchmarks, with basis sets ranging from $[\mathrm{S} / \mathrm{H}]$ [4s3p1d/2s1p] to [9s8p7d5f4g3h2i/7s6p5 d4f3g2h] have been employed to obtain the best technically possible value for the barrier to linearity of hydrogen sulphide. Following careful extrapolations of MP2, CCSD and CCSD(T) energies to the complete basis set (CBS) limit and inclusion of small corrections due to scalar relativistic terms, core polarization and core correlation effects, and the diagonal Born-Oppenheimer correction (DBOC), the final electronic (vibrationless) extrapolated barrier height of this study is $24423 \pm 75 \mathrm{~cm}^{-1}$. (C) 2000 Elsevier Science B.V. All rights reserved.
\end{abstract}

\section{Introduction}

The ground-state potential energy (hyper)surface (PES) of water has traditionally been a testing ground for the myriad theoretical approaches for computing (ro)vibrational eigenstates, resulting in a large number of publications on local (anharmonic force field) and (semi)global surfaces [1-6]. The most recent studies, based partially on state-of-the-art ab initio approaches $[1,3,4,6,7]$, resulted in PESs of unusually high accuracy. The best surfaces interpolate and to some extent extrapolate excellently. These studies have also shown that even small physical effects, readily neglected during most ab initio constructions of PESs, such as relativistic phenomena $[3,4]$ and

\footnotetext{
* Corresponding author. Tel.: +36-1-209-0555/1631; fax: +36-1-209-0602; e-mail: csaszar@para.chem.elte.hu
}

non-Born-Oppenheimer terms [1], may produce changes on the order of a few $\mathrm{cm}^{-1}$ for the rovibrational eigenstates of water.

The ground electronic state PES of hydrogen sulphide, $\mathrm{H}_{2} \mathrm{~S}$, the congener of $\mathrm{H}_{2} \mathrm{O}$, has also received considerable attention during the 1990's [818]. This interest is due to drastically increased spectroscopic capabilities for detecting higher-lying stretching and bending states of $\mathrm{H}_{2} \mathrm{~S}$ and of its isotopomers [15], to subsequent questions of normal mode vs. local mode character of vibrational overtones [16], to publicity over the four-fold clustering effects of high- $J$ rotational lines [10], and to the important role the $\mathrm{H}_{2} \mathrm{~S}$ molecule plays in the physics and chemistry of the interstellar medium and the atmospheres of planets and cool stars. Recently, two of us have been engaged [17] in the determination of a ground-state PES and dipole-moment surface (DMS) for $\mathrm{H}_{2} \mathrm{~S}$ comparable in accuracy to those of 
water. This Letter reports on the first results of this concerted study, which are of special relevance to the precise determination of the barrier to linearity on the ground-state PES of $\mathrm{H}_{2} \mathrm{~S}$.

The available semiglobal analytical representations of the ground-state PES of $\mathrm{H}_{2} \mathrm{~S}$ contain some extrapolation into regions of no or limited experimental data, such as the region around linearity. Table 1 summarizes geometric parameters of the bent and linear forms of $\mathrm{H}_{2} \mathrm{~S}$ and barriers to linearity available from the literature, which can be contrasted to our own high-level theoretical data generated during the course of this study. As expected, and confirmed at all levels of theory applied, $r(\mathrm{~S}-\mathrm{H})$ in the linear form of $\mathrm{H}_{2} \mathrm{~S}$ is shorter than the equilibrium $r(\mathrm{~S}-\mathrm{H})$ bond distance. Nevertheless, all published PESs for $\mathrm{H}_{2} \mathrm{~S}$ lack this feature; thus, they are inadequate for predicting higher-lying bending states. Sizable differences, ranging from $18792 \mathrm{~cm}^{-1}$ [8] to $31326 \mathrm{~cm}^{-1}$ [11], between values calculated from empirical and (semi)theoretical PESs can be observed for the barrier to linearity. Furthermore, as Table 1 and Fig. 1 demonstrate, farther away from equilibrium the different PES representations behave rather differently, and the otherwise high-quality surfaces due to Polyansky, Jensen and Tennyson [8] (henceforth PJT) and Senekowitsch, Carter, Zilch, Werner, Handy and Rosmus [13] (henceforth SCZWHR) show unphysical behavior as the HSH bond angle approaches small values. These problems and the fact that the barrier to linearity was shown to have a significant effect $[3,4]$ on the calculation of even low-lying bending states of water, calls for a careful, state-of-the-art ab initio quantum chemical investigation of the barrier to linearity of $\mathrm{H}_{2} \mathrm{~S}$. Relative energy predictions based on electronic structure calculations can have an accuracy [19] perhaps two orders of magnitude better than the spread in the aforementioned estimates for the barrier. Therefore, one goal of this study is to approach this ab initio limit as closely as technically possible. At the same time these calculations provide excellent guidelines concerning the level of theory needed for a converged $\mathrm{ab}$ initio representation of the lower-energy region of the ground-state PES of $\mathrm{H}_{2} \mathrm{~S}$ [17].

In order to arrive at the ab initio quantum chemical limit for energy differences such as the barrier to linearity of $\mathrm{H}_{2} \mathrm{~S}$, a systematic layout must be made

Table 1

Geometric parameters $^{\mathrm{a}}$ of the bent and linear forms and barrier to linearity (in $\mathrm{cm}^{-1}$ ) on the ground electronic state PES of hydrogen sulphide ${ }^{\mathrm{b}}$

\begin{tabular}{|c|c|c|c|c|c|}
\hline \multirow[t]{2}{*}{ Level } & \multicolumn{2}{|c|}{ Bent form } & \multirow{2}{*}{$\frac{\text { Linear form }}{r_{\mathrm{e}}(\mathrm{S}-\mathrm{H})}$} & \multirow[t]{2}{*}{ Barrier } & \multirow[t]{2}{*}{ Reference } \\
\hline & $r_{\mathrm{e}}(\mathrm{S}-\mathrm{H})$ & $\theta_{\mathrm{e}}(\mathrm{H}-\mathrm{S}-\mathrm{H})$ & & & \\
\hline cc-pVTZ CCSD(T)_all & 1.3376 & 92.277 & 1.3138 & 24522 & this work \\
\hline aug-cc-pVTZ CCSD(T)_all & 1.3375 & 92.012 & 1.3166 & 24268 & this work \\
\hline aug-cc-pVTZ CCSD(T)_FC & 1.3419 & 92.300 & 1.3197 & 24333 & this work \\
\hline aug-CVTZ CCSD(T)_all & 1.3350 & 92.236 & & & this work \\
\hline aug-cc-pVQZ CCSD(T)_all & 1.3373 & 92.295 & 1.3159 & 24221 & this work \\
\hline aug-cc-pVQZ CCSD(T)_FC & 1.3396 & 92.386 & 1.3184 & 24202 & this work \\
\hline PJT & 1.3360 & 92.331 & 1.3636 & 18792 & Ref. [8] \\
\hline $\mathrm{KJ}$ & 1.3366 & 92.226 & 1.3605 & 20867 & Ref. [10] \\
\hline $\mathrm{HC}$ & 1.3356 & 92.12 & 1.3731 & 31326 & Ref. [11] \\
\hline KH & 1.3356 & 92.12 & 1.3321 & 29498 & Ref. [12] \\
\hline SCZWHR & 1.3376 & 92.335 & 1.3397 & 22588 & Ref. [13] \\
\hline BZWRR SCF & 1.3271 & 94.162 & 1.4027 & 27656 & Ref. [9] \\
\hline BZWRR CEPA & 1.3355 & 92.246 & 1.4486 & 23311 & Ref. [9] \\
\hline BZWRR Corr. CEPA & 1.3356 & 92.12 & 1.4480 & 21980 & Ref. [9] \\
\hline Exp. & 1.3356 & 92.11 & & & Ref. [14] \\
\hline
\end{tabular}

\footnotetext{
${ }^{\mathrm{a}}$ Distances $\left(r_{\mathrm{e}}\right)$ in ångstrom and angle $\left(\theta_{\mathrm{e}}\right)$ in degrees.
}

${ }^{\mathrm{b}}$ See text for description of basis sets and levels of theory. Under 'Level' the empirical and (semi)theoretical PESs are referenced by the initials of the authors of the corresponding paper. '_all' stands for an all-electron calculation, while '_FC' stands for a frozen-core calculation. 


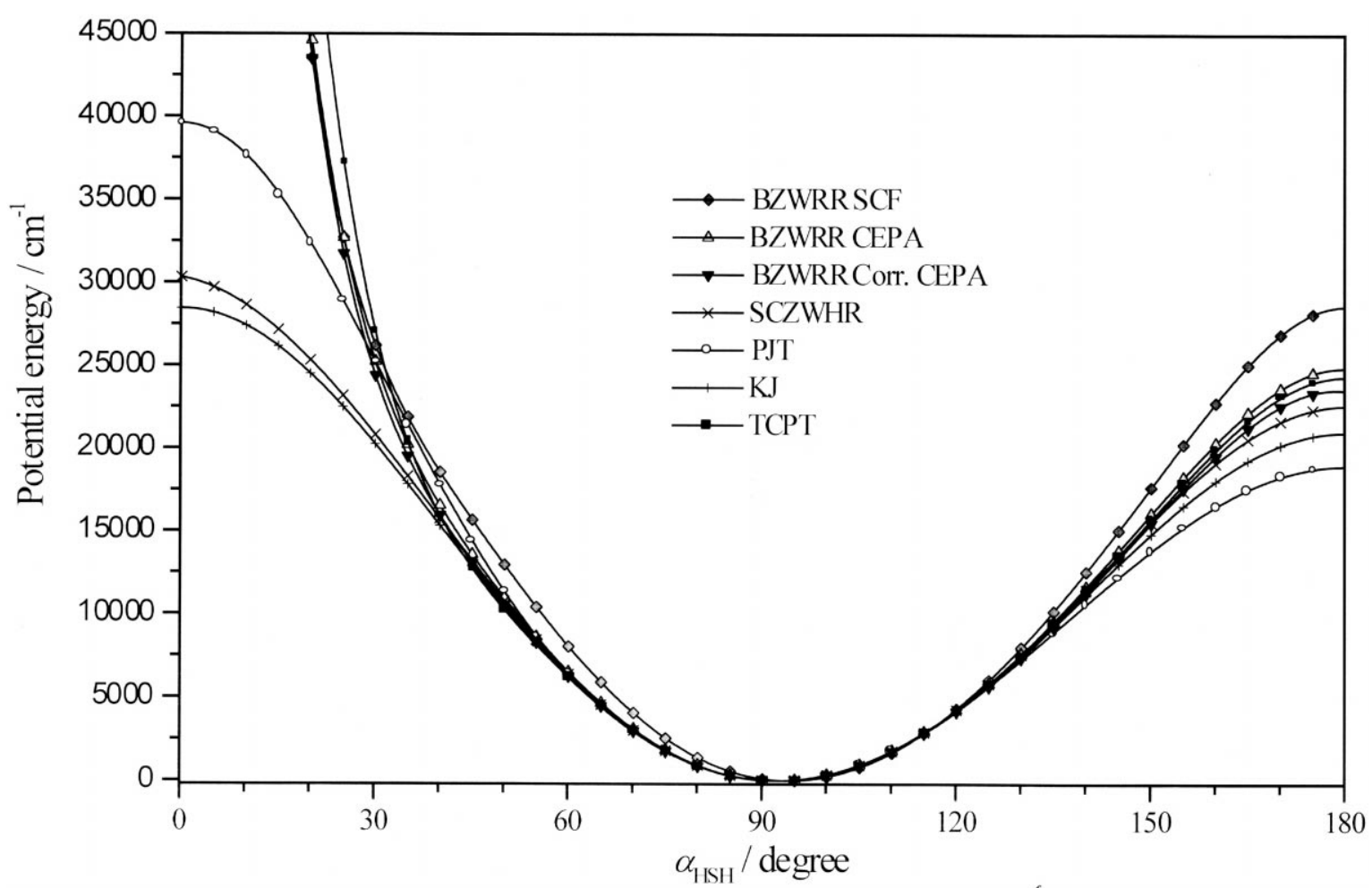

Fig. 1. Behavior of different PESs (BZWRR, Ref. [9]; SCZWHR, Ref. [13]; PJT, Ref. [8]; KJ, Ref. [10]; TCPT, Ref. [17]) of H ${ }_{2}$ S under bending of the molecule while fixing the $\mathrm{S}-\mathrm{H}$ bond lengths at their equilibrium value.

of the dual basis set and electron correlation convergence, including physically motivated extrapolations to the complete basis set (CBS) and full configuration interaction (FCI) asymptotes [19]. The best ab initio predictions include corrections for core correlation and special relativity, as well as the diagonal Born-Oppenheimer correction (DBOC). For the problem of the $\mathrm{H}_{2} \mathrm{~S}$ barrier, the present study pushes the application of sophisticated ab initio methodologies to new heights. Obviously, the ab initio calculations result directly in estimates of the electronic (vibrationless) barrier height. These values can directly be compared to the best empirical and semitheoretical (semi)global PESs, which are also complete-dimensional, vibrationless surfaces.

\section{Theoretical methods}

The electronic structure computations reported in this paper have been performed with the ACES II
[20], PSI [21,22], Gaussian-94 [23], DIRCCR12-95

[24] and DALTON [25] program systems.

Reference electronic wave functions have been determined by the single-configuration restricted Hartree-Fock (RHF) method [26]. Dynamical electron correlation was accounted for by standard methods [26] of electronic structure theory: Møller-Plesset (MP) perturbation theory from second through fifth order (MP2-MP5), the coupled cluster (CC) method including all single and double (CCSD) and triple excitations (CCSDT), and configuration interaction (CI) computations (CISD, CISDT, CISDTQ, CISDTQP, CISDTQPH and FCI). The CCSD(T) method, which estimates the effect of connected triple excitations through a perturbative term, was employed extensively. The Brueckner doubles (BD) method with perturbational estimates for both connected triple and quadruple excitations [BD(TQ)] was also employed. Extrapolation of the MP $n$ series, resulting in $\mathrm{MP} \infty$, was performed via shifted [2,1] Padé approximants when fifth-order energies were 
Table 2

Valence focal-point analysis of the inversion barrier of hydrogen sulphide ${ }^{\mathrm{a}}$

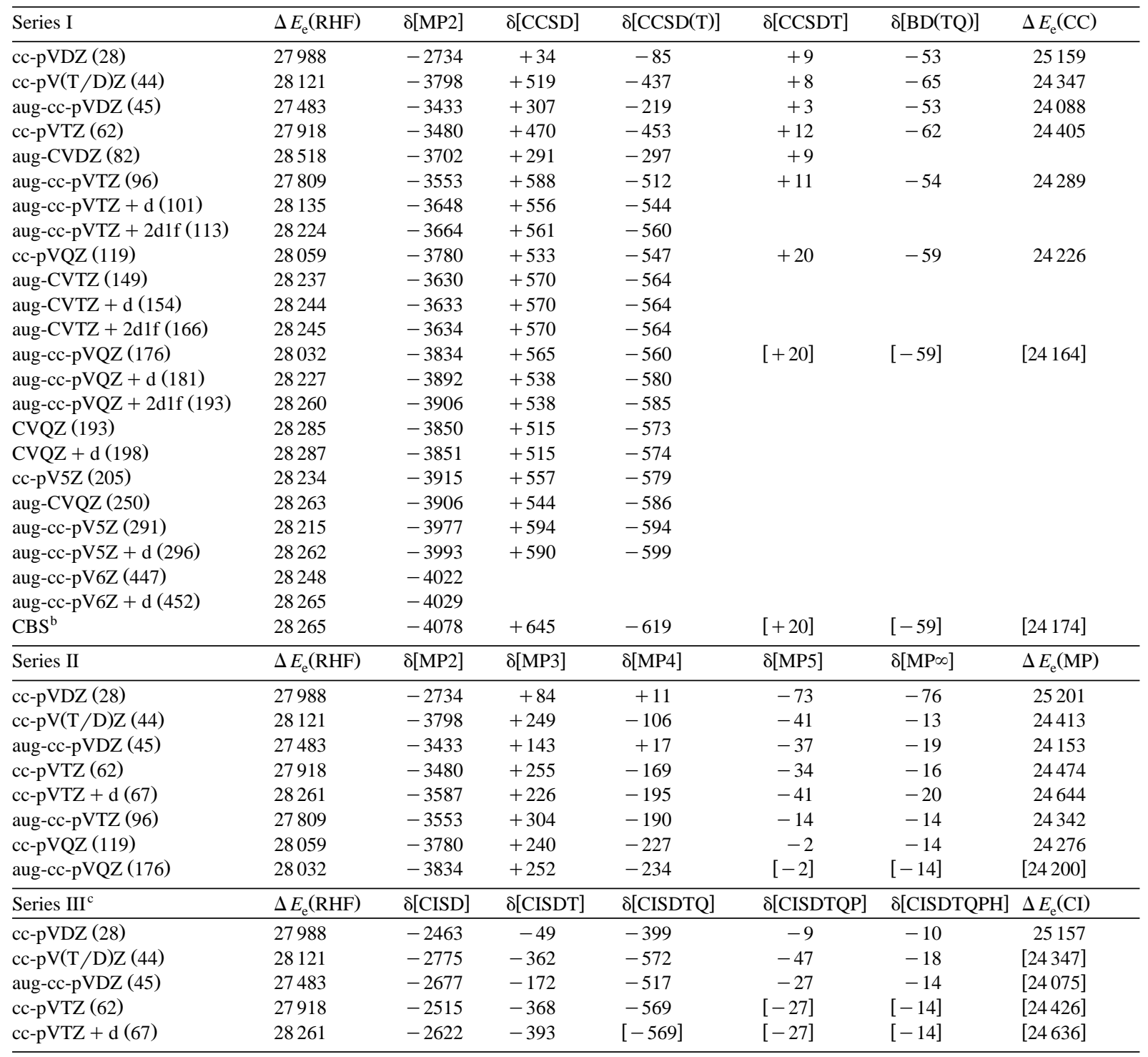

${ }^{a}$ For each basis set the total number of contracted Gaussian functions is given in parentheses. For correlated-level calculations the symbol $\delta$ denotes the increment in the relative energy $\left(\Delta E_{\mathrm{e}}\right)$ with respect to the preceding level of theory as given by the hierarchy $\mathrm{RHF} \rightarrow \mathrm{MP} 2 \rightarrow \mathrm{CCSD} \rightarrow \mathrm{CCSD}(\mathrm{T}) \rightarrow \mathrm{CCSDT} \rightarrow \mathrm{BD}(\mathrm{TQ}), \mathrm{RHF} \rightarrow \mathrm{MP} 2 \rightarrow \mathrm{MP} 3 \rightarrow \mathrm{MP} 4 \rightarrow \mathrm{MP} 5 \rightarrow \mathrm{MP} \infty$ and $\mathrm{RHF} \rightarrow \mathrm{CISD} \rightarrow \mathrm{CISDT} \rightarrow$ CISDTQ $\rightarrow$ CISDTQP $\rightarrow$ CISDTQPH $\rightarrow$ FullCI, for Series I, Series II and Series III, respectively. Brackets signify assumed increments from smaller basis set results. All values are given in $\mathrm{cm}^{-1}$.

${ }^{\mathrm{b}}$ The complete basis set (CBS) RHF barrier is obtained by extrapolation of aug-cc-pV(Q,5,6)Z $+\mathrm{d}$ energies. The CBS correlation increments are obtained by extrapolation of aug-cc-pV\{5,6\}Z $+\mathrm{d}$ results for MP2 and aug-cc-pV\{Q,5\}Z $+\mathrm{d}$ results for CCSD and CCSD(T).

${ }^{\mathrm{c}}$ For the bent structure, the largest computed CI energies, for each basis set, have been determined employing 19604169 (cc-pVDZ), 320548825 [cc-pV(T/D)Z], 379564739 (aug-cc-pVDZ) and 28085271 (cc-pVTZ) determinants appropriately. For the cc-pVDZ basis set the difference between the FCI and CISDTQPH barriers is $-0.1 \mathrm{~cm}^{-1}$. For all basis sets this increment has been used to arrive at the FCI limit. 
available. In valence-only correlated-level calculations (designated as $1 \mathrm{~s} 2 \mathrm{~s} 2 \mathrm{p}$ ), the $1 \mathrm{~s}, 2 \mathrm{~s}$ and $2 \mathrm{p}$ core orbitals of $\mathrm{S}$ were kept doubly occupied, while in the so-called 1s calculations only the $1 \mathrm{~s}$ core orbitals were kept doubly occupied. No virtual molecular orbitals were frozen in any of the correlation treatments.

The basis sets chosen for the calculations include the correlation-consistent (aug)-cc-pVXZ [X= 2(D), 3(T), 4(Q), 5, 6] families of basis sets developed by Dunning and co-workers [27,28]. Basis sets designed to describe the core region adequately [7], denoted as (aug)-cc-pCVXZ for first-row atoms [27,28], are not available for sulphur from Ref. [28]. To construct such basis sets, we completely uncontracted the aug-cc-pVXZ basis sets for $\mathrm{H}$ and $\mathrm{S}$ and then augmented the sulphur sets with tight $\{2 \mathrm{~d}, 2 \mathrm{~d} 2 \mathrm{f}, 2 \mathrm{~d} 2 \mathrm{f} 2 \mathrm{~g}\}$ sets for $X=\{\mathrm{D}, \mathrm{T}, \mathrm{Q}\}$, appropriately, whose exponents were obtained by even-tempered extension into the core with a geometric ratio of 3 . The resulting basis sets are denoted here simply as aug-CVXZ $(X=\{\mathrm{D}, \mathrm{T}, \mathrm{Q}\})$. Although core polarization $[7,29]$ plays no important role in determining the barrier height of $\mathrm{SiH}_{3}^{-}$[30], in this study the correlation-consistent basis sets have been augmented by tight $d$ and $f$-functions, whose exponents were obtained by an even-tempered extension into the core with a geometric ratio of 3. The resulting basis sets, aimed to account for the core polarization effect, are denoted by their conventional name with suffixes $+\mathrm{d}$ (one added $d$-function) and $+2 \mathrm{~d} 1 \mathrm{f}$ (two added $d$ and one added $f$-function).
Reference geometries, $r_{\mathrm{e}}(\mathrm{S}-\mathrm{H})=1.33730$ (1.31593) $\AA$ and $\theta_{\mathrm{e}}(\mathrm{H}-\mathrm{S}-\mathrm{H})=92.295(180.0)^{\circ}$ in the $\mathrm{C}_{2 v}\left(\mathrm{D}_{\circ \mathrm{h}}\right)$ cases, for the single-point energy and energy correction calculations have been obtained at the all-electron aug-cc-pVQZ $\operatorname{CCSD}(\mathrm{T})$ level. The results of further geometry optimizations for the bent and linear structures are summarized in Table 1. The $\mathrm{C}_{2 v}$ geometric parameters compare favorably with available empirical values [14], namely $r_{\mathrm{e}}(\mathrm{S}-\mathrm{H})=$ $1.3356 \AA$ and $\theta_{\mathrm{e}}(\mathrm{H}-\mathrm{S}-\mathrm{H})=92.11^{\circ}$ (cf. Table 1$)$. The effects of relativistic and DBOC energy corrections on the bond length of the linear structure have been investigated. The all-electron cc-pVTZ $\operatorname{CCSD}(\mathrm{T})$ bond length, $1.31383 \AA$, changes to $1.31310 \AA$ when the entire DPT energy correction of about $-1.11 E_{\mathrm{h}}$ is added to the cc-pVTZ $\operatorname{CCSD}(\mathrm{T})$ energies of about $-398.86 E_{\mathrm{h}}$. The DBOC energy correction, calculated at the TZ2Pf + diff RHF level (see Section 6 below), changes the same non-relativistic optimum bond length to $1.31350 \AA$.

The entire valence ab initio analysis of the barrier to linearity is laid out in Table 2 . One can gauge the effect of using fixed geometries in the valence focal-point scheme by comparing the aug-cc-pVTZ CCSD(T)_FC barriers given in Tables 1 and 2, since in Table 1 the barrier (column 5) refers to optimized reference structures, while in Table 2 all barriers have been obtained at fixed all-electron aug-cc-pVQZ $\operatorname{CCSD}(\mathrm{T})$ geometries. The difference between the two barriers is only $1.2 \mathrm{~cm}^{-1}$, providing clear support for fixing the reference structures during the focal-point analysis.

Table 3

Contribution (in $\mathrm{cm}^{-1}$ ) of core correlation to the inversion barrier of hydrogen sulphide ${ }^{\mathrm{a}}$

\begin{tabular}{|c|c|c|c|c|c|c|}
\hline \multirow[t]{2}{*}{ Basis } & \multicolumn{2}{|l|}{ MP2 } & \multicolumn{2}{|c|}{ CCSD } & \multicolumn{2}{|c|}{$\operatorname{CCSD}(\mathrm{T})$} \\
\hline & $1 \mathrm{~s}$ & all & $1 \mathrm{~s}$ & all & $1 \mathrm{~s}$ & all \\
\hline aug-CVDZ (82) & -145 & -154 & +72 & +65 & +51 & +43 \\
\hline aug-CVTZ (149) & -144 & -153 & +56 & +48 & +28 & +21 \\
\hline aug-CVTZ + d (154) & -183 & -199 & +20 & +6 & -6 & -19 \\
\hline aug-CVTZ + 2d1f (166) & -183 & -203 & +23 & +6 & -3 & -20 \\
\hline CVQZ (193) & -173 & -187 & +29 & +18 & +1 & -10 \\
\hline CVQZ + d (198) & -186 & & +17 & & -10 & \\
\hline aug-CVQZ (250) & -172 & -186 & +31 & +20 & +3 & -8 \\
\hline
\end{tabular}

\footnotetext{
${ }^{a}$ For each basis set the total number of contracted Gaussian functions is given in parentheses. 'all' refers to the difference between a frozen-core (1s, $2 \mathrm{~s}$ and $2 \mathrm{p}$ on $\mathrm{S}$ ) and an all-electron calculation, while '1s' refers to the difference between a frozen-core (1s, $2 \mathrm{~s}$ and $2 \mathrm{p}$ on $\mathrm{S})$ and a frozen $1 \mathrm{~s}$ (on $\mathrm{S}$ ) calculation.
} 
Table 4

Relativistic corrections to the inversion barrier of hydrogen sulphide ${ }^{\mathrm{a}}$

\begin{tabular}{|c|c|c|c|c|c|c|c|c|c|c|c|c|c|c|c|c|c|c|c|c|c|c|}
\hline \multirow[t]{3}{*}{ Basis } & \multicolumn{6}{|l|}{ RHF } & \multicolumn{5}{|l|}{ MP2 } & \multicolumn{5}{|l|}{ CCSD } & \multicolumn{6}{|l|}{$\operatorname{CCSD}(\mathrm{T})$} \\
\hline & \multicolumn{2}{|l|}{$\overline{\mathrm{D} 1}$} & \multirow[t]{2}{*}{ D2 } & \multirow[t]{2}{*}{ MV } & \multirow[t]{2}{*}{$\Delta \mathrm{DPT}$} & \multirow[t]{2}{*}{ Sum } & \multirow[t]{2}{*}{$\overline{\mathrm{D} 1}$} & \multirow[t]{2}{*}{$\mathrm{D} 2$} & \multirow[t]{2}{*}{ MV } & \multirow[t]{2}{*}{$\Delta \mathrm{DPT}$} & \multirow[t]{2}{*}{ Sum } & \multirow[t]{2}{*}{$\overline{\mathrm{D} 1}$} & \multirow[t]{2}{*}{ D2 } & \multirow[t]{2}{*}{$2 \mathrm{MV}$} & \multirow[t]{2}{*}{$\Delta \mathrm{DPT}$} & \multirow[t]{2}{*}{ Sum } & \multicolumn{2}{|l|}{ D1 } & \multirow[t]{2}{*}{ D2 } & \multirow[t]{2}{*}{ MV } & \multirow[t]{2}{*}{$\Delta \mathrm{DPT}$} & \multirow[t]{2}{*}{ Sum } \\
\hline & $\bar{S}$ & $2 \mathrm{H}$ & & & & & & & & & & & & & & & $\overline{\mathrm{s}}$ & $2 \mathrm{H}$ & & & & \\
\hline cc-pVDZ(28) & $\{-608.9\}$ & & 4.5 & 808.8 & 1.0 & 205.3 & -611.2 & 4.9 & 819.7 & 2.8 & 216.2 & -628.0 & 5.1 & 844.8 & 3.2 & 225.1 & $\{-626.1\}$ & & 5.2 & 843.0 & 3.3 & 225.4 \\
\hline aug-cc-pVDZ(45) & $\{-588.6\}$ & & 4.1 & 775.6 & -0.2 & 190.9 & -567.9 & 4.5 & 761.3 & 2.3 & 200.2 & -602.1 & 5.0 & 796.9 & 10.5 & 210.2 & $\{-586.2\}$ & & 4.8 & 789.2 & 2.2 & 210.0 \\
\hline cc-pVTZ(62) & -615.5 & -2.7 & 4.5 & 798.4 & 11.5 & 196.4 & -602.4 & 4.7 & 792.9 & 9.6 & 204.3 & -618.0 & 4.8 & 814.2 & 10.1 & 211.1 & -611.1 & -2.2 & 4.9 & 810.3 & 9.6 & 211.8 \\
\hline CVDZ(65) & -680.9 & & 5.3 & 908.2 & -0.1 & 232.5 & -697.7 & 5.6 & 940.1 & 0.0 & 248.0 & -713.9 & 5.8 & 965.4 & 0.0 & 257.3 & $\{-710.8\}$ & & 5.8 & 962.5 & 0.0 & 257.6 \\
\hline aug-CVDZ(82) & $\{-654.0\}$ & & 5.1 & 871.4 & 0.0 & 222.5 & -645.6 & 5.3 & 872.5 & 0.0 & 232.1 & -668.8 & 5.5 & 906.6 & 0.0 & 243.3 & $\{-662.3\}$ & & 5.6 & 899.1 & 0.0 & 242 \\
\hline aug-cc-pVTZ(96) & $\{-604.2\}$ & & 4.5 & 785.6 & 11.8 & 197.7 & -584.7 & 4.8 & 773.3 & 9.9 & 203.4 & -602.1 & 5.0 & 796.9 & 10.5 & 210.2 & $\{-595.3\}$ & & 5.0 & 790.5 & 10.1 & 210.4 \\
\hline CVTZ(115) & -669.3 & & 5.1 & 888.4 & -0.1 & 224.2 & -662.5 & 5.2 & 890.6 & 0.0 & 233.4 & -677.1 & 5.4 & 912.2 & 0.0 & 240.4 & $\{-671.2\}$ & & 5.4 & 906.4 & -0.1 & 240.7 \\
\hline cc-pVQZ(119) & -585.6 & -2.9 & 4.3 & 745.3 & 22.7 & 183.5 & -573.9 & 4.5 & 744.1 & 18.8 & 193.5 & -589.6 & 4.7 & 765.1 & 19.4 & 199.5 & -581.5 & -2.3 & 4.7 & 760.7 & 18.9 & 200.5 \\
\hline aug-CVTZ(149) & $\{-660.5\}$ & & 5.1 & 876.5 & 0.2 & 221.2 & -646.9 & 5.1 & 869.9 & 0.0 & 228.2 & -663.8 & 5.3 & 894.1 & 0.0 & 235.5 & $\{-655.9\}$ & & 5.3 & 885.9 & 0.1 & 235.4 \\
\hline $\begin{array}{l}\text { aug-CVTZ } \\
+\mathrm{d}(154)\end{array}$ & $\{-660.5\}$ & & & 876.6 & & & & & & & & & & & & & $\{-656.5\}$ & & & 886.3 & & \\
\hline $\begin{array}{l}\text { aug-CVTZ } \\
+2 \mathrm{~d} 1 \mathrm{f}(166)\end{array}$ & $\{-660.5\}$ & & & 876.6 & & & & & & & & & & & & & $\{-656.4\}$ & & & 886.2 & & \\
\hline CVQZ (193) & $\{-666.4\}$ & & & 883.2 & & & -654.1 & 5.2 & 878.1 & 0.0 & 229.2 & -669.0 & 5.3 & 899.5 & 0.0 & 235.7 & $\{-661.5\}$ & & & 891.7 & & \\
\hline cc-pV5Z(205) & -626.2 & -3.0 & 4.78 & 816.9 & & & & & & & & & & & & & -628.1 & -2.4 & 5.9 & 835.8 & & \\
\hline aug-CVQZ(250) & $\{-663.6\}$ & & & 879.5 & & & -648.4 & 5.1 & 870.7 & 0.0 & 227.4 & -664.8 & 5.3 & 893.9 & 0.0 & 234.3 & & & & & & \\
\hline
\end{tabular}

${ }^{a}$ Obtained with all electrons correlated. All values are given in $\mathrm{cm}^{-1}$. D1 = one-electron Darwin term; D2 = two-electron Darwin term; MV = one-electron mass-velocity term; $\Delta \mathrm{DPT}=$ correction term arising in the lowest order of DPT; $\mathrm{Sum}=\mathrm{MV}+\mathrm{D} 1+\mathrm{D} 2+\Delta \mathrm{DPT}$ (see text for details). The D1 values given in braces correspond to the sum of the atomic terms. 
Table 5

Absolute DBOC corrections at RHF level at the bent and linear geometries and relative energy contributions to the barrier to linearity of $\mathrm{H}_{2}{ }^{32} \mathrm{~S}^{\mathrm{a}}$

\begin{tabular}{llll}
\hline Basis & Bent & Linear & Contrib. \\
\hline cc-pVDZ (28) & 1345.6 & 1367.9 & +22.3 \\
DZP (33) & 1348.9 & 1371.9 & +23.0 \\
DZP + (39) & 1349.2 & 1368.3 & +19.1 \\
aug-cc-pVDZ (45) & 1342.8 & 1368.3 & +25.5 \\
TZ2P (49) & 1345.5 & 1376.2 & +30.7 \\
TZ2P+ (55) & 1345.7 & 1378.1 & +32.4 \\
cc-pVTZ (62) & 1347.0 & 1373.1 & +26.1 \\
TZ2Pf (66) & 1347.4 & 1372.8 & +25.4 \\
TZ2Pf + (72) & 1347.5 & 1374.9 & +27.4
\end{tabular}

${ }^{\mathrm{a}}$ See text for basis set details. All values are in $\mathrm{cm}^{-1}$.

Auxiliary corrections to the barrier height due to core correlation, relativistic effects and the DBOC term are collected in Tables 3-5.

\section{Valence ab initio limits}

Data for the valence focal-point analysis [19] of the barrier to linearity of $\mathrm{H}_{2} \mathrm{~S}$ are collected in Table 2. Three $n$-particle series have been investigated: coupled-cluster theory (CC; Series I), Møller-Plesset perturbation theory (MP; Series II), and configuration interaction theory (CI; Series III). The most balanced treatments are afforded by $\mathrm{CC}$ wave functions. The data obtained reveal the following about the barrier to linearity of $\mathrm{H}_{2} \mathrm{~S}$ and its $\mathrm{ab}$ initio determination:

(1) As observed repeatedly for relative energy changes on a single PES [19], upon enlargement of the one-particle basis set the most dramatic changes appear at the RHF and especially the MP2 levels. Augmentation of the basis with diffuse or core polarization functions has no substantial effect past MP2 (double excitation). Nevertheless, as observed repeatedly (see, e.g., Ref. [19]), inclusion of diffuse functions in the basis accelerates the convergence of the energetic results significantly.

(2) Augmentation of the aug-cc-pVXZ basis sets with just a single tight $d$-function (core polarization [29]) improves drastically the convergence characteristics of the energy results obtained for $\mathrm{H}_{2} \mathrm{~S}$ at the
RHF (and to a much lesser extent at the MP2) level. For example, the RHF barrier changes more than 300 $\mathrm{cm}^{-1}$ by inclusion of a single $d$-function into the aug-cc-pVTZ basis. The improved result is less than $100 \mathrm{~cm}^{-1}$ from the inferred RHF limit, a typical result observed for first-row elements [19]. Inclusion of a $+2 \mathrm{~d} 1 \mathrm{f}$ inner polarization set improves the RHF energetic results further, especially for $X=3$. While at the $\operatorname{CCSD}(\mathrm{T})$ level the barriers obtained with the original aug-cc-pVXZ basis sets $(\{24332,24203,24238\}$ with $\{X=3,4,5\})$ are closer to the corresponding CBS CCSD(T) limit of 24213 $\mathrm{cm}^{-1}$, the convergence characteristic of the $+\mathrm{d}$ series is better.

(3) $\Delta E_{\mathrm{e}}$ (RHF) and the $\delta[\mathrm{MP} 2]$ increment do not converge within even a modest $0.1 \mathrm{kcal} \mathrm{mol}^{-1}$ accuracy until the conventional correlation-consistent basis sets are extended drastically, well beyond the cc-pV6Z level. Addition of core polarization functions greatly improves the convergence of both $\Delta E_{\mathrm{e}}(\mathrm{RHF})$ and the $\delta[\mathrm{MP} 2]$ increment.

(4) Basis set extrapolations (see below) reveal that in the particular case of the barrier to linearity of $\mathrm{H}_{2} \mathrm{~S}$, MP2 theory, which includes an approximate treatment of double substitutions, works extremely well due to fortuitous error cancellations beyond this level.

(5) CCSD theory compensates partially for the MP2 contribution to the barrier. Furthermore, with all basis sets past aug-cc-pVDZ the $\delta$ [CCSD] and $\delta[C C S D(T)]$ increments have very similar magnitude and opposite sign, making their combined contribution almost negligible.

(6) The minuscule $\delta[C C S D T]$ increments in Table 2 testify that performance of the $\operatorname{CCSD}(\mathrm{T})$ method, which approximates perturbatively the energy contributions of connected triple excitations, is excellent compared to the full CCSDT treatment.

(7) The FCI and high-order CI results (Series III of Table 2) reveal that (a) $\delta[\mathrm{BD}(\mathrm{TQ})]$ approximates excellently the effect of quadruple excitation in CC theory; (b) the extrapolated CC barriers are considerably more dependable than the MP $\infty$ barrier heights; for example, using the aug-cc-pVDZ basis the extrapolated $\mathrm{CC}$ barrier agrees with the FCI result within $2 \mathrm{~cm}^{-1}$ but MPo is off by $44 \mathrm{~cm}^{-1}$.

Extrapolation of RHF and correlation energies to the complete basis set (CBS) limit has been achieved 
through the relations $[19,31-33] \quad E^{X}=E_{\mathrm{CBS}}+$ $a \exp (-b X)$ and $E^{X}=E_{\mathrm{CBS}}+c X^{-3}$, respectively, where $E_{\mathrm{CBS}}$ is the extrapolated energy, while $E^{X}$ denotes energies obtained from correlation-consistent-type basis sets with cardinal number $X$. It is clear that extrapolation of the original cc-pVXZ and aug-cc-pVXZ RHF energies is more or less meaningless. The extrapolated RHF energies, based on aug-cc-pV(Q,5,6)Z + d results, are -398.720235 and $-398.591449 E_{\mathrm{h}}$ at the bent and linear structures, respectively, yielding a limiting value of 28265 $\mathrm{cm}^{-1}$ for the RHF barrier. The total correlation energies determined with the two largest aug-cc$\mathrm{pVXZ}+\mathrm{d}$ basis sets at the MP2, CCSD and $\operatorname{CCSD}(\mathrm{T})$ levels of theory yield the following net barriers to linearity when the resulting $E_{\mathrm{CBS}}$ correlation components are appended to the extrapolated RHF limit: $\Delta E_{\mathrm{e}}\{\mathrm{MP} 2, \mathrm{CCSD}, \mathrm{CCSD}(\mathrm{T})\}=$ $\{24187,24832,24213\} \mathrm{cm}^{-1}$.

\section{Core correlation}

The results for the contribution of core correlation to the barrier to linearity of $\mathrm{H}_{2} \mathrm{~S}$ are collected in Table 3. Two sets of numbers have been obtained at each level of theory: in the $1 \mathrm{~s}$ case the sulphur $1 \mathrm{~s}$ core electrons have been kept frozen during the correlation treatment, while the $1 \mathrm{~s} 2 \mathrm{~s} 2 \mathrm{p}$ case refers to the difference between the all-electron and the traditional frozen-core calculation keeping the $1 \mathrm{~s}, 2 \mathrm{~s}$ and $2 p$ orbitals of sulphur doubly occupied.

It is clear from Table 3 that, unlike in the case of first-row molecular prototypes studied (e.g., water) [19], the core correlation contribution to the barrier to linearity is small. In fact, it has a much smaller effect on the barrier height than the core polarization effect, discussed above. The core correlation correction is rather sensitive to the level of theory applied for its calculation. In particular, MP2, with all basis sets studied, provides a correction which is much exaggerated. It is also notable that the $1 \mathrm{~s}$ and $1 \mathrm{~s} 2 \mathrm{~s} 2 \mathrm{p}$ numbers differ very little, suggesting that differential core correlation of the $1 \mathrm{~s}$ electrons of sulphur is small. The best estimate of the core correlation correction to the barrier to linearity of $\mathrm{H}_{2} \mathrm{~S}$ is -8 $\mathrm{cm}^{-1}$, obtained at the aug-CVQZ CCSD(T) level.

\section{Relativistic effects}

In this study, relativistic corrections to the electronic energy have been gauged by the lowest order of the direct perturbation theory (DPT) approach developed by Kutzelnigg $[34,35]$. For atoms lighter than argon, the DPT approach has been proved [35] to provide at least $99 \%$ of the relativistic energy correction even at its lowest order. Furthermore, the lowest-order DPT correction can be decomposed into the widely utilized mass-velocity (MV), Darwin (D) and spin-orbit (SO) terms plus a correction term $(\Delta \mathrm{DPT})$ due to basis set incompleteness [35]. In this study the one-electron MVD and the two-electron Darwin (D2) terms have been calculated by a slight modification of the DIRCCR12-95 package [24] following the recipe of Ref. [36]. The DPT approach has been incorporated into the ab initio package DALTON [25].

The relativistic results obtained at the RHF, MP2, CCSD and CCSD(T) levels of theory using several basis sets are given in Table 4. A few aspects of these data warrant comment:

(1) The energy of the hypothetical relativistic $S$ atom without electron correlation can be approximated as $\Delta E_{\text {rel }}(\mathrm{S})=2 w(1 \mathrm{~s}, 14)+2 w(2 \mathrm{~s}, 12)+$ $2 w\left(2 \mathrm{p}_{1 / 2}, 10\right)+4 w\left(2 \mathrm{p}_{3 / 2}, 10\right)$, where $w(\mathrm{AO}, Z)$ means the energy of an electron in atomic orbital AO $(\mathrm{AO}=1 \mathrm{~s}, 2 \mathrm{~s}, 2 \mathrm{p})$ in the effective field of a nuclear charge $Z$. Coupling this approximation with the usual [35] first-order relativistic perturbative correction for H-like atoms,

$w=\frac{-Z^{4} \alpha^{2}}{2 n^{4}}\left(\frac{n}{j+1 / 2}-\frac{3}{4}\right)$,

results in $\Delta E_{\text {rel }}(\mathrm{S})=-1.09 E_{\mathrm{h}}$. The relativistic correction determined in this study for $\mathrm{H}_{2} \mathrm{~S}$ is approximately $-1.11 E_{\mathrm{h}}$.

(2) The explicitly computed MV and D1 corrections are about -4.6 and $+3.5 E_{\mathrm{h}}$, respectively, canceling out most of their effect.

(3) The D2 correction term, which depends on the minuscule probability of two electrons being at the same point in space, is small, only $-0.03 E_{\mathrm{h}}$. This two-electron contribution is virtually identical in the 
linear and bent structures, making its effect on the barrier almost negligible $\left(5 \mathrm{~cm}^{-1}\right)$. Nevertheless, its magnitude is comparable to the electron correlation contribution to the relativistic effect $\left(10 \mathrm{~cm}^{-1}\right)$.

(4) The overall relativistic shift on the barrier is not particularly sensitive to the level of theory, all reasonable results reported in Table 4 lie between 200 and $240 \mathrm{~cm}^{-1}$. Our computations also show that the RHF shift, as expected, is affected substantially by the addition of core polarization and correlation functions to the basis set (aug-cc-pVTZ RHF vs. aug-CVTZ RHF). Additional extension of the augCVTZ basis with $+\mathrm{d}$ or $+2 \mathrm{~d} 1 \mathrm{f}$ sets, on the other hand, has no effect on the relativistic correction to the barrier.

(5) Basis set convergence of the $\triangle \mathrm{DPT}$ term is fast once high-exponent functions, those describing the core region effectively, are included in the basis set. A large $\triangle \mathrm{DPT}$ term suggests an inadequate one-electron basis.

(6) Neither the D2 nor the $\triangle \mathrm{DPT}$ terms are important for the dependable prediction of the relativistic contribution to the barrier.

Our final prediction based on data taken from Table 4 is that relativistic effects increase the inversion barrier of $\mathrm{H}_{2} \mathrm{~S}$ by about $+230 \mathrm{~cm}^{-1}$.

\section{The diagonal Born-Oppenheimer correction (DBOC)}

Computation of the mass-dependent diagonal Born-Oppenheimer correction (DBOC) was performed at the Hartree-Fock (HF) level within the formalism of Handy, Yamaguchi and Schaefer [37] and by means of the BORN program operating within the PSI package [21]. The results obtained for $\mathrm{H}_{2}{ }^{32} \mathrm{~S}$ are presented in Table 5. Standard and built-in basis sets for HF calculations, including DZP, TZ2P and TZ2Pf have been employed during computation of the DBOC correction. In some cases these basis sets have been augmented to include diffuse functions, indicated by + in Table 5 .

As expected, the DBOC corrections for the bent and linear forms of $\mathrm{H}_{2}{ }^{32} \mathrm{~S}$ are relatively large, about $6 \mathrm{~m} E_{\mathrm{h}}$. Nevertheless, the DBOC corrections at the two reference structures have about the same magni- tude; the net effect on the barrier to linearity of $\mathrm{H}_{2}{ }^{32} \mathrm{~S}$ changes only between +19 and $+32 \mathrm{~cm}^{-1}$. No clear convergence of the results is seen in Table 5 ; therefore, we take $+27 \mathrm{~cm}^{-1}$ as the best estimate of the DBOC correction, the result obtained at the TZ2Pf + RHF level.

\section{Net vibrationless barrier}

In conclusion, our final prediction for the vibrationless barrier to linearity of $\mathrm{H}_{2}{ }^{32} \mathrm{~S}$ is $(24174-8$ $+230+27)=24423 \mathrm{~cm}^{-1}$. This result is substantially higher than the values of $18792 \mathrm{~cm}^{-1}$ [8] and $21980 \mathrm{~cm}^{-1}$ [9], the best previous estimates. A conservative error estimate which can be attached to our calculated barrier is $\pm 75 \mathrm{~cm}^{-1}$. This value is based on valence-only $\{\mathrm{RHF}, \delta \mathrm{MP} 2, \delta \mathrm{CCSD}$, $\delta C C S D(T), \delta C C S D T, \delta B D(T Q)\}$ error estimates of $\{ \pm 2, \pm 25, \pm 15, \pm 10, \pm 5, \pm 3\} \mathrm{cm}^{-1}$, a joint estimate of $\pm 40 \mathrm{~cm}^{-1}$ for the core correlation, relativistic and DBOC corrections, and finally taking $3 / 4$ of the sum of these error estimates.

\section{Acknowledgements}

This work has been partially supported by the Hungarian Ministry of Culture and Education (FKFP 0117/1997), by the Scientific Research Foundation of Hungary (OTKA T024044 and T033074), by the Hungarian-British Joint Academic and Research Programme (project no. 076), and by the Stichting Academisch Rekencentrum Amsterdam (SARA). MLL was supported by Sandia National Laboratories. Sandia is a multiprogram laboratory operated by Sandia, a Lockheed Martin Company, for the United States Department of Energy under Contract DEAC04-94AL85000. The research of WK has been made possible by a fellowship of the Royal Netherlands Academy of Arts and Sciences. The authors would like to thank J. Tennyson, O.L. Polyansky and W.D. Allen for helpful discussions. Help of Y. Yamaguchi with the DBOC calculations is gratefully acknowledged. 


\section{References}

[1] N.F. Zobov, O.L. Polyansky, C.R. Le Sueur, J. Tennyson, Chem. Phys. Lett. 260 (1996) 381.

[2] O.L. Polyansky, P. Jensen, J. Tennyson, J. Chem. Phys. 105 (1996) 6490, and references therein.

[3] A.G. Császár, J.S. Kain, O.L. Polyansky, N.F. Zobov, J. Tennyson, Chem. Phys. Lett. 293 (1998) 317.

[4] A.G. Császár, J.S. Kain, O.L. Polyansky, N.F. Zobov, J. Tennyson, Chem. Phys. Lett. 312 (1999) 613, (E).

[5] G. Tarczay, A.G. Császár, W. Klopper, V. Szalay, W.D. Allen, H.F. Schaefer III, J. Chem. Phys. 110 (1999) 11971.

[6] H. Partridge, D.W. Schwenke, J. Chem. Phys. 106 (1997) 4618, and references therein.

[7] A.G. Császár, W.D. Allen, Y. Yamaguchi, H.F. Schaefer III, in: P. Jensen, P.R. Bunker (Eds.), Computational Molecular Spectroscopy, Wiley, Chichester, 2000.

[8] O.L. Polyansky, P. Jensen, J. Tennyson, J. Mol. Spectrosc. 178 (1996) 184, and references therein.

[9] P. Botschwina, A. Zilch, H.-J. Werner, P. Rosmus, E.A. Reinsch, J. Chem. Phys. 85 (1986) 5107.

[10] I. Kozin, P. Jensen, J. Mol. Spectrosc. 163 (1994) 483.

[11] L. Halonen, T. Carrington Jr., J. Chem. Phys. 88 (1988) 4171.

[12] E. Kauppi, L. Halonen, J. Phys. Chem. 94 (1990) 5779.

[13] J. Senekowitsch, S. Carter, A. Zilch, H.-J. Werner, N.C. Handy, P. Rosmus, J. Chem. Phys. 90 (1989) 783.

[14] R.L. Cook, F.C. DeLucia, P.J. Helminger, J. Mol. Struct. 28 (1975) 237.

[15] O.N. Ulenikov, E.A. Ditenberg, I.M. Olekhnovitsch, S. Alanko, M. Koivusaari, R. Anttila, J. Mol. Spectrosc. 191 (1998) 239, and references therein.

[16] O. Vaittinen, L. Biennier, A. Campargue, J.-M. Flaud, L. Halonen, J. Mol. Spectrosc. 184 (1997) 288, and references therein.

[17] G. Tarczay, A.G. Császár, O.L. Polyansky, J. Tennyson, to be published.

[18] S. Miller, J. Tennyson, P. Rosmus, J. Senekowitsch, I.M. Mills, J. Mol. Spectrosc. 143 (1990) 61.

[19] A.G. Császár, W.D. Allen, H.F. Schaefer III, J. Chem. Phys. 108 (1998) 9751.
[20] J.F. Stanton, J. Gauss, J.D. Watts, W.J. Lauderdale, R.J Bartlett, Int. J. Quant. Chem., Quant. Chem. Symp. 26 (1992) 897.

[21] C.L. Janssen et al., PSI 2.0.8, PSITECH, Watkinsville, GA, 1994.

[22] T.D. Crawford et al., PSI 3.0, PSITECH, Watkinsville, GA 30677, USA, 1999.

[23] M.J. Frisch et al., Gaussian-94, Revision B.2, Gaussian, Pittsburgh, PA, 1995.

[24] W. Klopper, J. Noga, J. Chem. Phys. 103 (1995) 6127.

[25] T. Helgaker et al., DALTON, an electronic structure program, Release 1.0, 1997.

[26] W.J. Hehre, L. Radom, P.v.R. Schleyer, J.A. Pople, Ab Initio Molecular Orbital Theory, Wiley-Interscience, New York, 1986 and references therein.

[27] T.H. Dunning Jr., J. Chem. Phys. 90 (1989) 1007.

[28] Extensible Computational Chemistry Environment Basis Set Database, Version 1.0, as developed and distributed by the Molecular Science Computing Facility, Environmental and Molecular Sciences Laboratory, which is part of the Pacific Northwest Laboratory, P.O. Box 999, Richland, WA 99352, U.S.A. and funded by the U.S. Department of Energy. The Pacific Northwest Laboratory is a multi-program laboratory operated by Battelle Memorial Institute for the U.S. Department of Energy under contract DE-AC06-76RLO 1830.

[29] J.M.L. Martin, J. Chem. Phys. 108 (1998) 2791, and references therein.

[30] K. Aarset, A.G. Császár, W. Klopper, W.D. Allen, H.F. Schaefer III, J. Noga, J. Chem. Phys. 112 (2000) 4053.

[31] D. Feller, J. Chem. Phys. 96 (1992) 6104.

[32] D. Feller, J. Chem. Phys. 98 (1993) 7059.

[33] A. Halkier, T. Helgaker, P. Jørgensen, W. Klopper, H. Koch, J. Olsen, A.K. Wilson, Chem. Phys. Lett. 286 (1998) 243.

[34] W. Kutzelnigg, E. Ottschofski, R. Franke, J. Chem. Phys. 102 (1995) 1740.

[35] E. Ottschofski, W. Kutzelnigg, J. Chem. Phys. 102 (1995) 1752.

[36] W. Klopper, J. Comp. Chem. 18 (1997) 20.

[37] N.C. Handy, Y. Yamaguchi, H.F. Schaefer III, J. Chem. Phys. 84 (1986) 4481. 